\title{
A Note on Some Wishart Expectations ${ }^{1}$
}

\author{
By R. J. Muirhead ${ }^{2}$
}

Summary: In a recent paper Sharma and Krishnamoorthy (1984) used a complicated decisiontheoretic argument to derive an identity involving expectations taken with respect to the Wishart distribution $W_{m}(n, I)$. A more general result, proved using an elementary moment generating function argument, and some applications, are given in this paper.

\section{An Expectation Identity}

Let the random $m \times m$ matrix $S$ have the Wishart distribution $W_{m}(n, \Sigma)$, with probability density function

$$
c_{m, n}(\operatorname{det} \Sigma)^{-n / 2} \exp \left(-1 / 2 \operatorname{tr} \Sigma^{-1} S\right)(\operatorname{det} S)^{1 / 2(n-m-1)}, \quad \begin{gathered}
S>0, \Sigma>0, \\
0, n>m-1
\end{gathered}
$$

where

$$
c_{m, n}^{-1}=2^{m n / 2} \Gamma_{m}(1 / 2 n),
$$

with

$$
\Gamma_{m}(a)=\pi^{1 / 4 m(m-1)} \prod_{i=1}^{m} \Gamma(a-1 / 2(i-1)) .
$$

Using an innovative, but rather complicated and involved decision-theoretic argument, Sharma and Krishnamoorthy (1984) proved that when $\Sigma=I_{m}$,

$$
E\left[(\operatorname{tr} S)^{2} \operatorname{tr}\left(S^{\alpha}\right)\right]=(m n+2+2 \alpha) E\left[\operatorname{tr} S \operatorname{tr}\left(S^{\alpha}\right)\right],
$$

an identity which holds for all $\alpha$ for which the expectations exist. Here we give an elementary proof of a more general result which yields (1) as a special case. The general result is given in the following theorem.

1 This work was supported by the National Science Foundation.

2 Prof. Robb J. Muirhead, Department of Statistics, University of Michigan, 419 South State Street, Ann Arbor, Michigan 48109-1027, USA. 
Theorem: Suppose that $S \sim W_{m}(n, \Sigma)$. Let $h(S)$ be a real-valued measurable function of $S$ such that the function $f(t ; S)=h\left(t^{-1} S\right), t>0$, is differentiable at $t=1$. Let $f^{\prime}(t ; S)=\frac{\partial}{\partial t} f(t ; S)$. Then

$$
E\left[\operatorname{tr}\left(\Sigma^{-1} S\right) h(S)\right]=m n E[h(S)]-2 E\left[f^{\prime}(1 ; S)\right]
$$

provided the expectations involved exist.

Proof: For $t>0$ define the function $g(t)$ as

$$
g(t)=c_{m, n}(\operatorname{det} \Sigma)^{-n / 2} t^{m n / 2} \int_{S>0} \exp \left(-\frac{t}{2} \operatorname{tr} \Sigma^{-1} S\right)(\operatorname{det} S)^{1 / 2(n-m-1)} h(S) d S,
$$

and note that $g(1)=E[h(S)]$.

Differentiating (3) with respect to $t$ (justified by dominated convergence provided $E\left[\operatorname{tr}\left(\Sigma^{-1} S\right) h(S)\right]$ exists) and putting $t=1$ gives

$$
g^{\prime}(1)=1 / 2 m n E[h(S)]-1 / 2 E\left[\operatorname{tr}\left(\Sigma^{-1} S\right) h(S)\right] .
$$

Now put $X=t S$ in (3); then $g(t)$ can be written alternatively as

$$
g(t)=c_{m, n}(\operatorname{det} \Sigma)^{-n / 2} \int_{X>0} \exp \left(-1 / 2 \operatorname{tr} \Sigma^{-1} X\right)(\operatorname{det} X)^{1 / 2(n-m-1)} f(t ; X) d X,
$$

from which it follows that

$$
g^{\prime}(1)=E\left[f^{\prime}(1 ; S)\right]
$$

Equating (4) and (5) gives the desired result (2) and completes the proof.

In many interesting applications the function $h(\cdot)$ has the property that, for $x>0, h(x S)=x^{l} h(S)$ for some real $l$. Then $f(t ; S)=h\left(t^{-1} S\right)=t^{-l} h(S)$, so that

$$
f^{\prime}(1 ; S)=-\ln (S) \text {. }
$$

This yields the following result.

Corollary: If $h(x S)=x^{l} h(S)$ for some $l$ then

$$
E\left[\operatorname{tr}\left(\Sigma^{-1} S\right) h(S)\right]=(m n+2 l) E[h(S)] .
$$

The identity (1) of Sharma and Krishnamoorthy (1984) follows immediately from (6) by taking $\Sigma=I$ and $h(S)=\operatorname{tr} S \operatorname{tr}\left(S^{\alpha}\right)$, so that $l=\alpha+1$. Another identity, used by 
Efron and Morris (1976) in the context of decision-theoretic estimation of $\Sigma^{-1}$, is

$$
E\left[\frac{\operatorname{tr} \Sigma^{-1} S}{\operatorname{tr} S}\right]=(m n-2) E\left[\frac{1}{\operatorname{tr} S}\right] ;
$$

this is a special case of $(6)$ with $h(S)=(\operatorname{tr} S)^{-1}$, so that $l=-1$.

\section{Applications}

Many interesting expectations can be evaluated using (6). Some of these, in which $k$ and $r$ are nonnegative integers and

$$
(a)_{k}=a(a+1) \ldots(a+k-1),
$$

are:

$$
\begin{aligned}
& E\left[\left(\operatorname{tr} \Sigma^{-1} S\right)^{k}\right]=2^{k}\left({ }^{1} / 2 m n\right)_{k}, \\
& E\left[\left(\operatorname{tr} \Sigma^{-1} S\right)^{-k}\right]=(-1 / 2)^{k} /(-1 / 2 m n+1)_{k}, \quad(2 k<m n) \\
& E\left[\left(\operatorname{tr} \Sigma^{-1} S\right)^{k} \operatorname{tr} S\right]=2^{k}(1 / 2 m n+1)_{k} n \operatorname{tr} \Sigma, \\
& E\left[\left(\operatorname{tr} \Sigma^{-1} S\right)^{k} \operatorname{tr} S^{-1}\right]=2^{k}(1 / 2 m n-1)_{k} \operatorname{tr} \Sigma^{-1} /(n-m-1), \quad(n>m+1) \\
& E\left[\left(\operatorname{tr} \Sigma^{-1} S\right)^{k} \operatorname{tr} \Sigma S^{-1}\right]=2^{k}(1 / 2 m n-1)_{k} m /(n-m-1), \quad(n>m+1) \\
& E\left[\left(\operatorname{tr} \Sigma^{-1} S\right)^{k}(\operatorname{det} S)^{r}\right]=2^{m r+k}(1 / 2 m n+r m)_{k} \frac{\Gamma_{m}(1 / 2 n+r)}{\Gamma_{m}(1 / 2 n)} \cdot(\operatorname{det} \Sigma)^{r} .
\end{aligned}
$$

These may all be derived using essentially similar arguments and known elementary properties of Wishart matrices. For example, (7) is proved in the following way. Put $h(S)=\operatorname{tr}\left(\Sigma^{-1 / 2} S \Sigma^{-1 / 2}\right)=\operatorname{tr}\left(\Sigma^{-1} S\right)$ in $(6)$, so that $l=1$. This gives

$$
E\left[\left(\operatorname{tr} \Sigma^{-1} S\right)^{2}\right]=(m n+2) E\left[\operatorname{tr}\left(\Sigma^{-1} S\right)\right]=(m n+2) m n
$$

where we have used the fact that $E(S)=n \Sigma$. Next, taking $h(S)=\left(\operatorname{tr} \Sigma^{-1} S\right)^{2}$, with $l=2$, gives

$$
E\left[\left(\operatorname{tr} \Sigma^{-1} S\right)^{3}\right]=(m n+4) E\left[\left(\operatorname{tr} \Sigma^{-1} S\right)^{2}\right]=(m n+4)(m n+2) m n .
$$

The result (7) for arbitrary $k$ follows trivially by induction. To prove (8) note that taking $h(S)=1 / \operatorname{tr} \Sigma^{-1} S$ in $(6)$ gives $(m n-2) E\left[\frac{1}{\operatorname{tr} \Sigma^{-1} S}\right]=1$. 
Next, taking $h(S)=\left(\operatorname{tr} \Sigma^{-1} S\right)^{-2}$ in (6) gives

$$
(m n-4) E\left[\left(\operatorname{tr} \Sigma^{-1} S\right)^{-2}\right]=E\left[\left(\operatorname{tr} \Sigma^{-1} S\right)^{-1}\right]=1 /(m n-2),
$$

and the rest of the argument is obvious. The proofs of the other identities are similar. Note that to derive (10), (11), and (12) we need the known results

$$
\begin{aligned}
& E\left(S^{-1}\right)=\frac{1}{n-m-1} \Sigma^{-1}, \\
& E\left[(\operatorname{det} S)^{r}\right]=2^{m r} \frac{\Gamma_{m}(1 / 2 n+r)}{\Gamma_{m}(1 / 2 n)}(\operatorname{det} \Sigma)^{r} .
\end{aligned}
$$

We conclude by giving two expectations involving zonal polynomials. Let $C_{k}(S)$ denote the zonal polynomial of $S$ corresponding to the partition $k=\left(k_{1}, k_{2}, \ldots, k_{m}\right)$ of the integer $k\left(k_{1} \geqslant k_{2} \geqslant \ldots \geqslant k_{m} \geqslant 0\right)$ (see e.g. James 1964 or Muirhead 1982, Chapter 7), and let

$$
(a)_{K}=\prod_{i=1}^{m}(a-1 / 2(i-1))_{k_{i}}
$$

The following expectations, in which $B$ is a non-random $m x m$ symmetric matrix, hold:

$$
\begin{aligned}
& E\left[\left(\operatorname{tr} \Sigma^{-1} S\right)^{r} C_{K}(S B)\right]=(1 / 2 m n+k)_{r} 2^{k+r}(1 / 2 n)_{k} C_{K}(B \Sigma), \\
& E\left[\left(\operatorname{tr} \Sigma^{-1} S\right)^{-r} C_{K}(S B)\right]=\frac{(-1)^{r} 2^{k-r}}{(-1 / 2 m n-k+1)_{r}}(1 / 2 n)_{K} C_{K}(B \Sigma) \quad(r<1 / 2 m n+k) .
\end{aligned}
$$

These may be derived from (6) in a similar way to (7)-(12) using the known result that

$$
E\left[C_{k}(S B)\right]=2^{k}(1 / 2 n)_{\kappa} C_{\kappa}(B \Sigma)
$$

(see e.g. Muirhead 1982, p. 251). 


\section{References}

Efron B, Morris C (1976) Multivariate empirical Bayes and estimation of covariance matrices. Ann Statist $4: 22-32$

James AT (1964) Distributions of matrix variates and latent roots derived from normal samples. Ann Math Statist 35:475-501

Muirhead RJ (1982) Aspects of multivariate statistical theory. John Wiley \& Sons, New York

Sharma D, Krishnamoorthy K (1984) An identity involving a Wishart matrix. To appear in Metrika

Received July 10, 1984

(Revised version August 6,1984) 